\title{
Graphene-based one-dimensional photonic crystal
}

\author{
Oleg L. Berman and Roman Ya. Kezerashvili \\ Physics Department, New York City College of Technology, \\ The City University of New York, \\ Brooklyn, NY 11201, USA \\ and \\ The Graduate School and University Center, \\ The City University of New York, \\ New York, NY 10016, USA
}

\begin{abstract}
A novel type of one-dimensional (1D) photonic crystal formed by the array of periodically located stacks of alternating graphene and dielectric stripes embedded into a background dielectric medium is proposed. The wave equation for the electromagnetic wave propagating in such a structure is solved in the framework of the Kronig-Penney model. The frequency band structure of 1D graphene-based photonic crystal is obtained analytically as a function of the filling factor and the thickness of the dielectric between graphene stripes. The photonic frequency corresponding to the electromagnetic wave localized by the defect of photonic crystal formed by the extra dielectric placed on the place of the stack of alternating graphene and dielectric stripes is obtained.

PACS numbers: 42.70.Qs, 78.67.Wj, 78.67.-n, 78.67.Pt
\end{abstract}

\section{INTRODUCTION}

Photonic crystals are formed by structures with the dielectric constant periodically varying in space [1]. Electromagnetic waves in photonic crystals have a band spectrum and a coordinate dependence caused by this periodicity of the dielectric constant. Interestingly enough that the solution of Maxwell's equations with a periodic dielectric constant, resulting in photonic band-gap structures, is similar to the solution to Schrödinger's equation for a periodic potential, resulting in the electron energy band-gap structures in solids. Electromagnetic waves penetrate in the photonic crystal similar to the Bloch waves of electrons in a regular crystal. The width of the photonic band gap depends on the geometrical parameters of the photonic crystal and the contrast of the dielectric constants of the constituent elements [2, 3]. Different materials have been used for the corresponding constituent elements including dielectrics, semiconductors and metals [2, 3, 6- 9]. The photonic crystals with superconducting elements have been studied in Refs. [10 16]. The one-dimensional photonic crystals formed by semiconductors were analyzed in Ref. [17]. It is well known that the properties of photonic crystals provide an opportunity to manipulate the emission, propagation and distribution of light [4, 5] and photonic crystals can be used as frequency filters. The properties of photonic crystals were reviewed in Ref. [18].

Photonic crystals are different from the regular solid crystals in the following way. While Schrödinger's equation describes the regular solid crystals via the scalar wave function, Maxwell's equations for photonic band-gap crystals describes the electric or magnetic field, which is a vector corresponding to the transverse electromagnetic waves.

A novel type of 2D electron system was experimentally obtained in graphene, which is a 2D honeycomb lattice of the carbon atoms that form the basic planar structure in graphite [19, 20]. Due to unusual properties of the band structure, electronic properties of graphene became the object of many recent experimental and theoretical studies 19 25. Graphene is a gapless semiconductor with massless electrons and holes which have been described as Diracfermions [26]. The unique electronic properties of graphene in a magnetic field have been studied recently [27 30]. The space-time dispersion of graphene conductivity was analyzed in Ref. [31]. A graphene-based two-dimensional photonic crystal was proposed in Ref. [32], where its frequency band structure was studied. Different types of 2D photonic crystals were reviewed in Ref. [33].

In this Paper, we consider a one-dimensional photonic crystal formed by an array of periodically located parallel stacks of alternating graphene and dielectric stripes embedding into a background dielectric medium. The graphene stripes are placed one under the other with the dielectric stripes placed between them. We calculate the frequency band structure of such a photonic crystal. The photonic band structure usually can be obtained using numerical calculations. In this Paper we obtain the analytical solution for the wave equation with the periodical dielectric function. We also calculate the frequency corresponding to the electromagnetic wave localized due to a defect in the array of the stacks of graphene stripes separated by a dielectric stripes.

The Paper is organized in the following way. In Sec. II we obtain the photonic band structure of the 1D graphenebased photonic crystal. In Sec. III we find the frequency corresponding to the electromagnetic wave localized by the defect of a 1D graphene-based photonic crystal. Finally, the discussion of the results and conclusions follow in Sec. IV. 


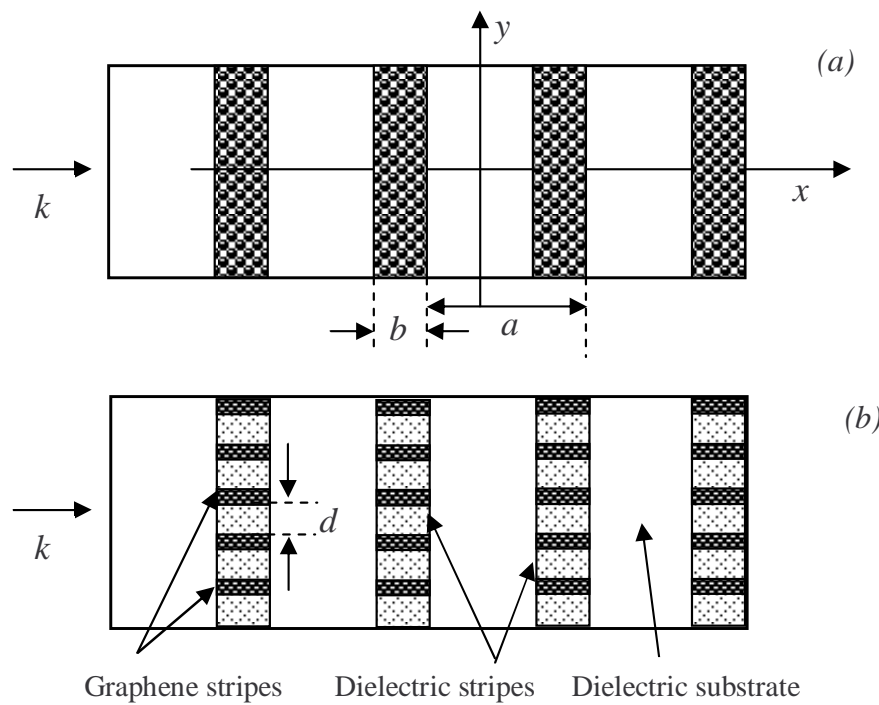

FIG. 1: The 1D photonic crystal with graphene stripes. (a) the top view; (b) the side view. The material of the dielectric stripes between graphene stripes can be the same as the material of the dielectric substrate.

\section{THE WAVE EQUATION FOR 1D PHOTONIC CRYSTAL WITH GRAPHENE STRIPES}

We consider polarized electromagnetic waves with the electric field $\mathbf{E}$ perpendicular to the plane of graphene stripes. The wave equation for the electric field in a dielectric media has the form [34]

$$
\Delta \mathbf{E}(\mathbf{r}, t)-\frac{\varepsilon(\mathbf{r}, t)}{c^{2}} \frac{\partial^{2} \mathbf{E}(\mathbf{r}, t)}{\partial t^{2}}=0
$$

where $\varepsilon(\mathbf{r}, t)$ is the dielectric constant of the media, and $c$ is the speed of light in vacuum. Looking for solutions with harmonic time variation of the electric field, i.e., $\mathbf{E}(\mathbf{r}, t)=\mathbf{E}(\mathbf{r}) e^{i \omega t}$, and considering the propagation of wave in the $x$-direction along the plane of graphene stripes and perpendicular to the graphene-dielectric boundaries one obtains from Eq. (1)

$$
\frac{\partial^{2} E_{z}(x)}{\partial x^{2}}+\frac{\omega^{2} \varepsilon(x, \omega)}{c^{2}} E_{z}(x)=0 .
$$

The dielectric constant of the $1 \mathrm{D}$ periodic structure is given by

$$
\varepsilon(x, \omega)=\left\{\begin{array}{l}
\varepsilon_{0}, \text { for }-\frac{1}{2}(a-b)+n a<x<\frac{1}{2}(a-b)+n a, \\
\varepsilon_{1}(\omega), \text { for } \frac{1}{2}(a-b)+n a<x<\frac{1}{2}(a+b)+n a,
\end{array}\right.
$$

where $\varepsilon_{0}$ is the dielectric constant of the dielectric, $\varepsilon_{1}(\omega)$ is the dielectric function of graphene multilayers separated by the dielectric barriers, $a$ is the period of $1 \mathrm{D}$ array of graphene stripes, $b$ is the width of graphene stripes, and $n$ is an integer. The 1D photonic crystal with graphene stripes separated by dielectric layers with a thickness $d$ is shown in Fig. 1. By introducing the filling factor $f$ the relation between $a$ and $b$ can be written as $b=a f$.

The dielectric function $\varepsilon_{1}(\omega)$ of a graphene multilayers system separated by dielectric layers with a dielectric constant $\varepsilon_{0}$ and a thickness $d$ is given by [35, 36]

$$
\varepsilon_{1}(\omega)=\varepsilon_{0}+\frac{4 \pi i \sigma_{g}(\omega)}{\omega d}
$$

where $\sigma_{g}(\omega)$ is the dynamical conductivity of doped graphene for the high frequencies $\left(\omega \gg k v_{F}, \omega \gg \tau^{-1}\right)$ at temperature $T$ given by [35, 36]

$$
\begin{aligned}
& \sigma_{g}(\omega)=\frac{e^{2}}{4 \hbar}[\eta(\hbar \omega-2 \mu) \\
& +\frac{i}{2 \pi}\left(\frac{16 k_{B} T}{\hbar \omega} \log \left[2 \cosh \left(\frac{\mu}{2 k_{B} T}\right)\right]\right. \\
& \left.\left.-\log \frac{(\hbar \omega+2 \mu)^{2}}{(\hbar \omega-2 \mu)^{2}+\left(2 k_{B} T\right)^{2}}\right)\right]
\end{aligned}
$$


Here $e$ is the charge of an electron, $\tau^{-1}$ is the electron collision rate, $\eta$ is the Heaviside step function, $k_{B}$ is the Boltzmann constant, and $\mu$ is the the chemical potential determined by the electron concentration, which is controlled by the doping. The chemical potential can be calculated as $\mu=\left(\pi n_{0}\right)^{1 / 2} \hbar v_{F}$, where the electron concentration is given by $n_{0}=\left(\mu /\left(\hbar v_{F}\right)\right)^{2} / \pi$ and $v_{F}=10^{8} \mathrm{~cm} / \mathrm{s}$ is the Fermi velocity of electrons in graphene [36].

We solve the wave equation (2) to find the eigenfrequencies corresponding to the electromagnetic wave penetrating in the photonic crystal shown in Fig. 1. This wave equation is mathematically similar to the Schrödinger equation for an electron moving in a one-dimensional rectangular periodic potential barrier described by the Kronig-Penney model. The eigenenergies of the Schrödinger equation corresponding to the Kronig-Penney model presented in Eq. (A1) are given by [37]:

$$
\cos (k a)=\cosh (\beta b) \cos [\alpha a(1-f)]+\frac{\alpha^{2}-\beta^{2}}{2 \alpha \beta} \sinh (\beta b) \sin [\alpha a(1-f)],
$$

where the wave vector $k$ is in the range $0 \leq k \leq 2 \pi / a$, and $\alpha$ and $\beta$ are defined as

$$
\begin{aligned}
& \alpha=\frac{\sqrt{\varepsilon_{0}}}{c} \omega, \\
& \beta=\frac{\sqrt{\varepsilon_{1}(\omega)}}{c} \omega .
\end{aligned}
$$

Applying Eq. (6) , Eq. (5) can be written in a form

$$
\cos (k a)=\cosh \left(\sqrt{\varepsilon_{1}(\omega)} \frac{a f}{c} \omega\right) \cos \left[\sqrt{\varepsilon_{0}} a \frac{(1-f)}{c} \omega\right]+\frac{\varepsilon_{0}-\varepsilon_{1}(\omega)}{2 \sqrt{\varepsilon_{0} \varepsilon_{1}}} \sinh \left(\sqrt{\varepsilon_{1}(\omega)} \frac{a f}{c} \omega\right) \sin \left[\sqrt{\varepsilon_{0}} \frac{a(1-f)}{c} \omega\right] .
$$

The eigenfrequencies of the 1D photonic crystal as functions of the wave vector $k$ can be obtained by substituting the dielectric constant of the multilayer graphene given by Eq. (3) into Eq. (7). The solutions of Eq. (7) for $\omega$ as functions of $k$ provides the frequency band structure for 1D photonic crystal formed by the periodically located parallel stacks of alternating graphene and dielectric stripes embedded into a background dielectric medium and allow one to determine the photonic band gap $\Omega$. Eq. (7) shows that the photonic band gap depends on the filling factor $f$ and the period of $1 \mathrm{D}$ array of graphene stripes $a$, as well as on the thickness $d$ of the dielectric stripes that separate the graphene stripes. Let us mention that dependence of the photonic band gap on the thickness $d$ of the dielectric layer, which separates the graphene stripes, is presented in (7), since the dielectric function $\varepsilon_{1}(\omega)$ (3) of a graphene multilayers system separated by dielectric layers depends on $d$.

\section{LOCALIZATION OF THE ELECTROMAGNETIC WAVE ON THE DEFECT}

Let us consider a defect in the array of stacks of alternating graphene and dielectric stripes embedded into a background dielectric medium. This defect is formed by one empty space or "1D vacancy" due to the absence of a stack of alternating graphene and dielectric stripes in one place, where it should be placed due to the periodicity. This place is filled by the dielectric. This extra dielectric stipe contributes to the dielectric contrast that results by adding the term $-\omega^{2} / c^{2}\left(\varepsilon_{1}(\omega)-\varepsilon_{0}\right) \gamma\left(\left|x-x_{0}\right|\right) E_{z}(x)$ to the r.h.s. in Eq. (2). Here $\gamma\left(\left|x-x_{0}\right|\right)=1$ for $\left|x-x_{0}\right| \leq b$, and $\gamma\left(\left|x-x_{0}\right|\right)=0$ for $\left|x-x_{0}\right|>b$, where $x_{0}$ corresponds to the coordinate in the middle of $1 \mathrm{D}$ defect, which is the coordinate of the middle of the absent graphene stripe. As a result we obtain the wave equation for the electric field for 1D photonic crystal with the defect:

$$
\frac{\partial^{2} E_{z}(x)}{\partial x^{2}}+\frac{\omega^{2}}{c^{2}}\left(\varepsilon(x, \omega)-\left(\varepsilon_{1}(\omega)-\varepsilon_{0}\right) \gamma\left(\left|x-x_{0}\right|\right)\right) E_{z}(x)=0 .
$$

Eq. (8) describes the periodic array of stacks of alternating graphene and dielectric stripes with the defect formed by one stack of alternating graphene and dielectric stripes being absent.

We solve the wave equation (8) to find the eigenfrequency corresponding to the electromagnetic wave localized at the defect formed by a background dielectric medium due to the absence of the stack of alternating graphene and dielectric stripes in one place, where it should be placed due to the periodicity. This wave equation is similar to the Schrödinger equation describing the electron in the periodic potential energy in the presence of the potential energy of the defect placed at the point $x_{0}$ given in Appendix B. From the mapping of Klein-Gordon type equation given by Eq. (B6) the wave equation for the electric field in graphene-based photonic crystal has the following form

$$
-\frac{4 k_{0}^{2} c^{4}}{3} \frac{d^{2} E_{z}(x)}{d x^{2}}-2 \omega^{2} \Omega^{2}\left(\varepsilon_{1}(\omega)-\varepsilon_{0}\right) \gamma\left(\left|x-x_{0}\right|\right) E_{z}(x)=\left(\omega^{4}-\Omega^{4}\right) E_{z}(x),
$$


where $k_{0}=2 \pi / a$ is the vector of the $1 \mathrm{D}$ reciprocal lattice. This Klein-Gordon type equation has the eigenvalue $\omega^{4}-\Omega^{4}$. In Eq. (9) $\Omega$ is the width of the forbidden band (photonic gap) in the spectrum of the electromagnetic wave.

The electric field can be obtained by mapping Eqs. (B7) and (B9) that correspond to wavefunctions of the stationary states. The continuity of the electric field and its derivative in the points $x=x_{0}+b / 2$ and $x=x_{0}-b / 2$ result in the transcendental equation determining the spectrum of the even states:

$$
\sqrt{\omega^{2} \Omega^{2}\left(\varepsilon_{1}(\omega)-\varepsilon_{0}\right)^{2}-\omega^{4}+\Omega^{4}} \tan \left(\sqrt{\omega^{2} \Omega^{2}\left(\varepsilon_{1}(\omega)-\varepsilon_{0}\right)^{2}-\omega^{4}+\Omega^{4}} b^{2} / c^{2}\right)=\sqrt{\left|\omega^{4}-\Omega^{4}\right|},
$$

as well as of the odd states:

$$
\sqrt{\omega^{2} \Omega^{2}\left(\varepsilon_{1}(\omega)-\varepsilon_{0}\right)^{2}-\omega^{4}+\Omega^{4}} \cot \left(\sqrt{\omega^{2} \Omega^{2}\left(\varepsilon_{1}(\omega)-\varepsilon_{0}\right)^{2}-\omega^{4}+\Omega^{4}} b^{2} / c^{2}\right)=-\sqrt{\left|\omega^{4}-\Omega^{4}\right|} .
$$

Solving Eqs. (10) and (11) with respect to the frequency $\omega$, we obtain the frequency corresponding to the photonic mode localized by the defect.

\section{DISCUSSION}

In the calculations below we assume $n_{0}=10^{11} \mathrm{~cm}^{-2}$. For simplicity, we consider the same material for a background dielectric medium and dielectric stripes between the graphene stripes. As the dielectric material we consider $\mathrm{SiO}_{2}$ with the dielectric constant $\varepsilon_{0}=4.5$. Using Eq. (7) we calculated the band structure for $1 \mathrm{D}$ graphene-based photonic crystal. The results of the calculations of the dispersion relation of the photonic crystal are presented in Fig. 2 . The photonic band structure is calculated for different distances between graphene layers $d$. In our calculations we used the chemical potential for the electrons in graphene $\mu=3.525 \times 10^{-21} \mathrm{~J}$, temperature $T=300 \mathrm{~K}$, the period of the $1 \mathrm{D}$ graphene stripes array $a=25 \times 10^{-6} \mathrm{~m}$, and the filling factor $f=0.3927$. According to the results of our calculations, the photonic band structure almost does not depend on $\varepsilon_{0}$ due to the fact that $\varepsilon_{0} \ll\left|\varepsilon_{1}\right|$. The results of our calculations demonstrate the strong dependence of the photonic band structure on the thickness $d$ of the dielectric that separates the graphene stripes. At $d=1 \mathrm{~nm}$ and $d=5 \mathrm{~nm}$ the distance between the lower and the upper dispersion curves is larger in the middle than at the edges. At $d=3 \mathrm{~nm}$ and $d=10$ nm the distance between the lower and the upper dispersion curves is larger at the edges than in the middle.

Using Eqs. (10) and (11) we calculate frequencies corresponding to the electromagnetic wave localized by the defect in the photonic crystal formed due to the absence of a stack of alternating graphene and dielectric stripes in one place, where it should be placed due to the periodicity. For the photonic crystal with the defect at $d=1$ nm we have $\nu=1.79 \mathrm{THz}$, at $d=3 \mathrm{~nm}$ we have $\nu=1.85 \mathrm{THz}$, at $d=5 \mathrm{~nm}$ we have $\nu=3.91 \mathrm{THz}$, at $d=10 \mathrm{~nm}$ we have $\nu=3.62 \mathrm{THz}$. All these frequencies are located inside the photonic band gap. Let us mention that since our approach is based on the Luttinger-Kohn model [39, 40], the localized frequency $\nu$ does not depend on momentum. The frequency localized by the defect is just a constant in the photonic band structure, which can be controlled by the thickness $d$ of the dielectric stripes that separate the graphene stripes, as well as by the filling factor $f$ and the period of 1D array of graphene stripes $a$.

In conclusion, the graphene-based photonic crystal proposed in this paper is a novel type one-dimensional photonic crystal formed by the array of the stacks of alternating graphene and dielectric stripes embedded into a background dielectric medium. This system can be analyzed as a 1D photonic crystal and the corresponding wave equation for the electromagnetic wave propagating in such a structure can be solved in the framework of the Kronig-Penney model. The frequency band structure is obtained analytically as a function of the filling factor, the period of 1D array of graphene stripes and the thickness of the dielectric between the graphene stripes. We obtain the photonic frequency corresponding to the electromagnetic wave localized at the defect of a photonic crystal formed by a background dielectric medium due to the absence of the stack of alternating graphene and dielectric stripes in one place, where it should be placed due to the periodicity.

\section{Acknowledgments}

This research was supported by PSC CUNY grant: Award \# 64197-00 42.

\section{Appendix A: The Schrödinger equation with the periodic potential}

The wave equation (2) with the dielectric constant given by Eq. (3) can be mapped onto the Schrödinger equation for an electron in the $1 \mathrm{D}$ periodic potential function approximated by a rectangular potential barrier, used in the 

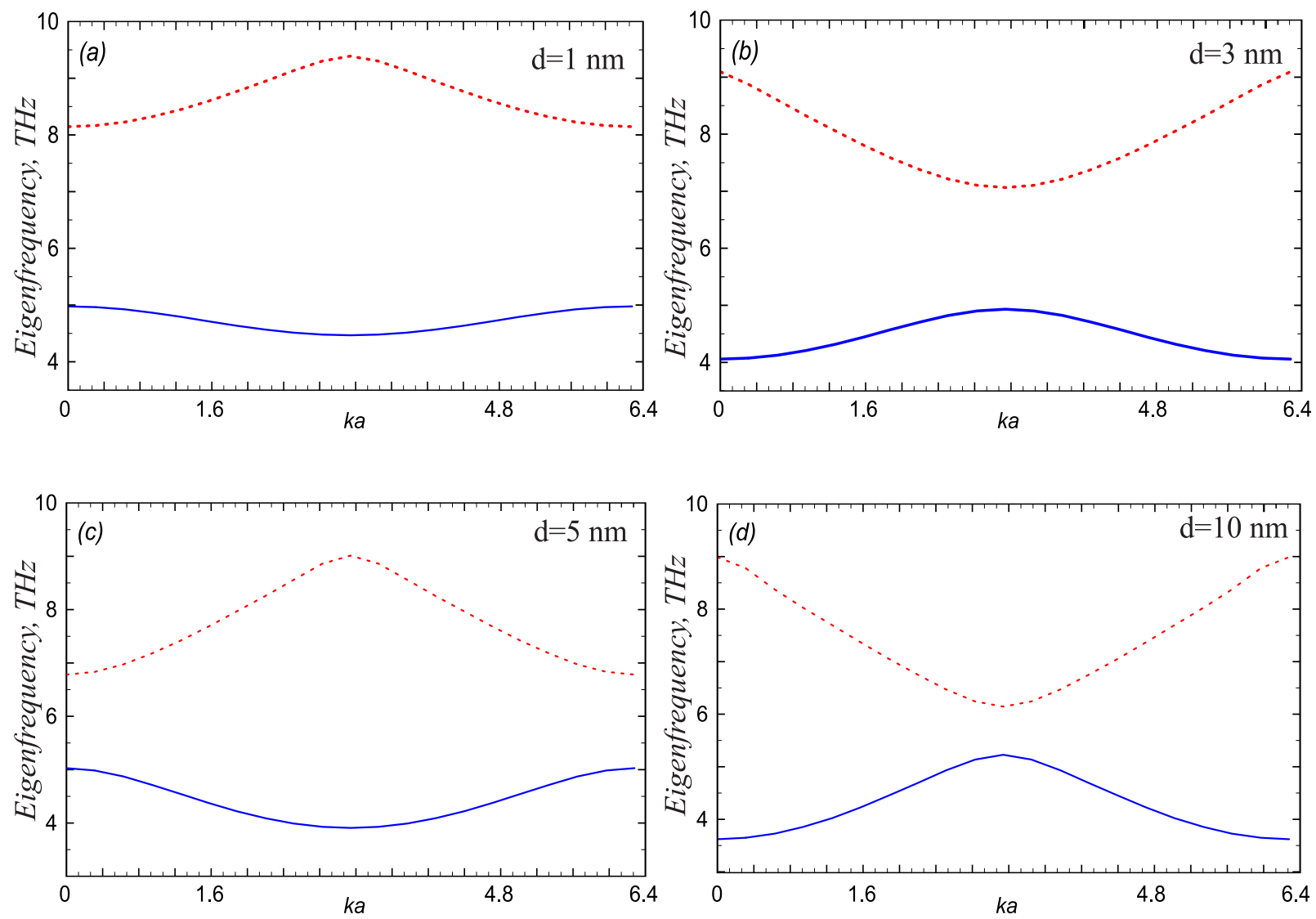

FIG. 2: The dispersion relation for the 1D graphene-based photonic crystal with $a=25 \mu \mathrm{m}$ and filling factor $f=0.3927$ for different thicknesses of the dielectric between graphene stripes.

Kronig-Penney model [37]:

$$
\begin{array}{rrrr}
-\frac{\hbar^{2}}{2 m_{0}} \frac{\partial^{2} \psi(x)}{\partial x^{2}} & =E \psi(x), & \text { for } \quad-\frac{1}{2}(a-b)+n a<x<\frac{1}{2}(a-b)+n a, \\
-\frac{\hbar^{2}}{2 m_{0}} \frac{\partial^{2} \psi(x)}{\partial x^{2}}+V(x) & =E \psi(x), V(x)=\frac{\hbar^{2} \omega^{2}}{2 m_{0} c^{2}} \varepsilon(x, \omega) & \text { for } \frac{1}{2}(a-b)+n a<x<\frac{1}{2}(a+b)+n a,
\end{array}
$$

where $m_{0}$ is the mass of an electron, $\psi(x)$ and $E$ are the wave function and energy of an electron, $V(x)$ is the potential due to the ion of the 1D crystal lattice approximated by a rectangular barrier, $a$ is the period of 1D array of the scatterers with the rectangular potential, $b$ is the width of the rectangular potential barrier, and $n$ is an integer.

It is easy to see that the wave equation (2) with the dielectric constant given by Eq. (3) can be mapped onto the Schrödinger equation (A1) for an electron in the 1D periodic potential corresponding to the Kronig-Penney model. The mapping relations are the following

$$
\begin{aligned}
\psi(x) & \equiv E_{z}(x) \\
\alpha^{2} & =\frac{2 m_{0}}{\hbar^{2}} E \equiv \frac{\varepsilon_{0}}{c^{2}} \omega^{2}, \\
\beta^{2} & =\frac{2 m_{0}}{\hbar^{2}}(E-V(x)) \equiv \frac{\varepsilon_{1}(\omega)}{c^{2}} \omega^{2} .
\end{aligned}
$$

The solution of Eq. (A1) is well known and is given by expression (5). 


\section{Appendix B: The Dirac-type equations for the the electromagnetic wave in the photonic crystal with the defect}

Following Ref. [15] we can map the wave equation for the photonic crystal with the defect onto the Schrödinger equation in the periodic field with the defect. After mapping of Eq. (8) onto the Schrödinger equation describing the electron with the effective electron mass $m_{0}$ in the periodic potential energy $V(x)$ in the presence of the potential energy of the defect $W \gamma\left(\left|x-x_{0}\right|\right)$ placed at the point $x_{0}$ we have

$$
\left[-\frac{\hbar^{2}}{2 m_{0}} \frac{d^{2}}{d x^{2}}+V(x)-W \gamma\left(\left|x-x_{0}\right|\right)\right] \psi(x)=\varepsilon_{\omega} \psi(x) .
$$

In Eq.(B1) $\psi(x)=E_{z}(x)$,

$$
\varepsilon_{\omega}=\frac{\hbar^{2} \omega^{2}}{2 m_{0} c^{2}}
$$

and the potential $V(x)$ is given in Eq. (A1) and potential $W$ is defined as

$$
W=\frac{\hbar^{2} \omega^{2}}{2 m_{0} c^{2}}\left(\varepsilon_{1}(\omega)-\varepsilon_{0}\right)
$$

Eq. (B1) has the same form as Eq. (6) in Ref. [38]. However, in our case the potential $W$ is defined by Eq. (B2) and corresponds to the potential of the defect in the Schrödinger equation for an "electron" in the periodic field of the crystal lattice and in the presence of the defect.

We will reduce the problem of the Schrödinger equation for a particle in the periodic potential $V(x)$ related to the system of the periodically placed stacks of alternating graphene and dielectric stripes embedded into a background dielectric medium and an "defect potential" $W$ related to a defect to a much simpler equation for the envelope wavefunctions. Taking into account the two-band structure and following the two-band model [38] we introduce a two-component spinor wave function $\psi(x)$

$$
\psi(x)=\left(\begin{array}{c}
\varphi(x) \\
\chi(x)
\end{array}\right)
$$

where two different neighboring bands are described by wave functions $\varphi(x)$ and $\chi(x)$. Note that Eq. (B1) contains both the periodic function $V(x)$ corresponding to the ideal lattice and $W$, describing the potential of a defect. Without a defect the energy spectrum would be described by two neighboring bands and the gap between them. Applying the standard two-band approach, we obtain an effective Dirac-type equation for the envelope spinor wave function, which implies the periodicity provided by $V(x)[15,38]$.

Note that Eq. (B1) describes an electron in the periodic potential of the ideal crystal lattice $V(x)$ and the potential of the defect $W$. If the solution corresponding to the absence of impurity $W=0$ is known, the energy levels of the electron localized by the defect can be obtained by replacing Eq. (B1) by the Dirac-type equation according to Luttinger-Kohn model described in Refs. 38 40]. This model implies a Dirac-type equation for the two-component spinor wave function. According to Ref. [38], the functions $\varphi_{n}(x)$ and $\chi_{n}(x)$ defined as

$$
\begin{aligned}
\varphi_{n}(x) & =\sum_{k} c_{n}(k) \exp [i k x / \hbar], \\
\chi_{n}(x) & =\sum_{k^{\prime}} d_{n}\left(k^{\prime}\right) \exp \left[i k^{\prime} x / \hbar\right]
\end{aligned}
$$

satisfy the set of the second order partial differential equations. Considering only two neighboring bands corresponding to the wave function $\psi(x)$ given by Eq. (B3) this set of equations for $\varphi_{n}(x)$ and $\chi_{n}(x)$ can be reduced to the Dirac-type equations for the two-component spinor (B3), which has the following form [38]

$$
\begin{aligned}
& {\left[\varepsilon_{\omega}-\Delta_{\omega}-W \gamma\left(\left|x-x_{0}\right|\right)\right] \varphi(x)+i \hbar s \sigma_{x} \frac{d \chi(x)}{d x}=0,} \\
& {\left[\varepsilon_{\omega}+\Delta_{\omega}-W \gamma\left(\left|x-x_{0}\right|\right)\right] \chi(x)+i \hbar s \sigma_{x} \frac{d \varphi(x)}{d x}=0,}
\end{aligned}
$$


where $s=\hbar k_{0} /\left(\sqrt{3} m_{0}\right)$. In Eq. (B5) $\sigma_{x}$ is Pauli matrix and $\Delta_{\omega}$ is the width of the forbidden band in the electron spectrum that, as it follows from the mapping of the wave equation for the electric field graphene-based photonic crystal onto Eq. (B1), is $\Delta_{\omega}=\frac{\hbar^{2} \Omega^{2}}{2 m_{0} c^{2}}$, where $\Omega$ is the width of the forbidden band (photonic gap) in the spectrum of the electromagnetic wave. Eqs. (B5) are obtained in the limit $\left|\varepsilon_{\omega}^{2}-\Delta_{\omega}^{2}\right| /\left(2 \Delta_{\omega}^{2}\right) \ll 1$. Defining the effective mass of a quasiparticle as $m_{\omega}=3 m_{0}^{2} \Delta_{\omega} /\left(\hbar^{2} k_{0}^{2}\right)$, and following the standard procedure of quantum electrodynamics [41, 42] we obtain from the system of Dirac-type Eqs. (B5) the following Klein-Gordon type equation. Note that the Klein Gordon equation can be reduced to a Schrödinger-like equation with an effective energy and an effective potential. If the potential is weak enough to ignore the $W^{2}$ term, the relativistic formalism becomes equivalent to the non-relativistic formalism. More importantly, in situations where the Klein-Gordon equation is not exactly solvable, the Schrödinger form of the Klein-Gordon equation sheds some light on the problem as it could be reduced to a solvable eigenvalue problem:

$$
\left[-\hbar^{2} s^{2} \frac{d^{2}}{d x^{2}}-2 m_{\omega} s^{2} W \gamma\left(\left|x-x_{0}\right|\right)\right] \Psi(x)=\left(\varepsilon_{\omega}^{2}-\Delta_{\omega}^{2}\right) \Psi(x) .
$$

This Klein-Gordon type equation has the form of the 2D Schrödinger equation for a particle in the square well potential well with the eigenvalue

$$
\mathcal{E}_{\omega}=\frac{\varepsilon_{\omega}^{2}-\Delta_{\omega}^{2}}{2 m_{\omega} s^{2}}
$$

The wavefunctions of the even stationary states have the form $\left(\mathcal{E}_{\omega}<0\right)$ :

$$
\begin{aligned}
& \Psi(x)=A_{1} \cos \left[\sqrt{2 m_{\omega}\left(W-\mathcal{E}_{\omega}\right) / \hbar^{2}} x\right], \quad\left|x-x_{0}\right| \leq b, \\
& \Psi(x)=B_{1} \exp \left[-\sqrt{2 m_{\omega} \mathcal{E}_{\omega} / \hbar^{2}} x\right], \quad\left|x-x_{0}\right|>b .
\end{aligned}
$$

The continuity of the wave function and its derivative in the points $x=x_{0}+b / 2$ and $x=x_{0}-b / 2$ result in the transcendental equation determining the spectrum of the even quantum states:

$$
\sqrt{W-\mathcal{E}_{\omega}} \tan \sqrt{2 m_{\omega}\left(W-\mathcal{E}_{\omega}\right) b^{2} / \hbar^{2}}=\sqrt{\left|\mathcal{E}_{\omega}\right|} .
$$

The wavefunctions of the odd stationary states have the form $\left(\mathcal{E}_{\omega}<0\right)$ :

$$
\begin{aligned}
& \Psi(x)=A_{2} \sin \left[\sqrt{2 m_{\omega}\left(W-\mathcal{E}_{\omega}\right) / \hbar^{2}} x\right], \quad\left|x-x_{0}\right| \leq b, \\
& \Psi(x)=B_{2} \exp \left[-\sqrt{2 m_{\omega} \mathcal{E}_{\omega} / \hbar^{2}} x\right], \quad\left|x-x_{0}\right|>b .
\end{aligned}
$$

The continuity of the wave function and its derivative in the points $x=x_{0}+b / 2$ and $x=x_{0}-b / 2$ result in the transcendental equation determining the spectrum of the odd quantum states:

$$
\sqrt{W-\mathcal{E}_{\omega}} \cot \sqrt{2 m_{\omega}\left(W-\mathcal{E}_{\omega}\right) b^{2} / \hbar^{2}}=-\sqrt{\left|\mathcal{E}_{\omega}\right|} .
$$

[1] E. Yablonovitch, Phys. Rev. Lett. 58, 2059 (1987).

[2] J. D. Joannopoulos, R. D. Meade, and J. N. Winn, Photonic Crystals: The Road from Theory to Practice (Princeton University Press, Princeton, NJ, 1995).

[3] J. D. Joannopoulos, S. G. Johnson, J. N. Winn, and R. D. Meade, Photonic Crystals: Molding the Flow of Light (Second Edition, Princeton University Press, Princeton, NJ, 2008).

[4] S. Y. Lin, J. Moreno, and J. G. Fleming, Appl. Phys. Lett. 83, 380 (2003).

[5] S. Y. Lin, J. G. Fleming, and I. EI-Kady, Appl. Phys. Lett. 83, 593 (2003).

[6] Z. Sun, Y. S. Jung, and H. K. Kim, Appl. Phys. Lett. 83, 3021 (2003).

[7] Z. Sun and H. K. Kim, Appl. Phys. Lett. 85, 642 (2004).

[8] A. R. McGurn and A. A. Maradudin, Phys. Rev. B 48, 17576 (1993). 
[9] V. Kuzmiak and A. A. Maradudin, Phys. Rev. B 55, 7427 (1997).

[10] H. Takeda and K. Yoshino, Phys. Rev. B 67, 073106 (2003).

[11] H. Takeda and K. Yoshino, Phys. Rev. B 67, 245109 (2003).

[12] H. Takeda, K. Yoshino, and A. A. Zakhidov, Phys. Rev. B 70, 085109 (2004).

[13] O. L. Berman, Yu. E. Lozovik, S. L. Eiderman, and R. D. Coalson, Phys. Rev. B 74, 092505 (2006).

[14] Yu. E. Lozovik, S. I. Eiderman, and M. Willander, Laser Physics 17, 1183 (2007).

[15] O. L. Berman, V. S. Boyko, R. Ya. Kezerashvili, and Yu. E. Lozovik, Phys. Rev. B 78, 094506 (2008).

[16] O. L. Berman, V. S. Boyko, R. Ya. Kezerashvili, and Yu. E. Lozovik, Laser Physics 19, 2035 (2009).

[17] B. Schulkin, L. Sztancsik, and J. F. Federici, Am. J. Phys. 72, 1051 (2004).

[18] K. Busch, G. von Freymann, S. Linden, S.F. Mingaleev, L. Tkeshelashvili, and M. Wegener, Phys. Reports 444, 101 (2007).

[19] K. S. Novoselov, A. K. Geim, S. V. Morozov, D. Jiang, Y. Zhang, S. V. Dubonos, I. V. Grigorieva, and A. A. Firsov, Science 306, 666 (2004).

[20] Y. Zhang, J. P. Small, M. E. S. Amori, and P. Kim, Phys. Rev. Lett. 94, 176803 (2005).

[21] K. S. Novoselov, A. K. Geim, S. V. Morozov, D. Jiang, M. I. Katsnelson, I. V. Grigorieva, and S. V. Dubonos, Nature (London) 438, 197 (2005).

[22] Y. Zhang, Y. Tan, H. L. Stormer, and P. Kim, Nature (London) 438, 201 (2005).

[23] K. Kechedzhi, O. Kashuba, and V. I. Fal'ko, Phys. Rev. B 77, 193403 (2008).

[24] M. I. Katsnelson, Europhys. Lett. 84, 37001 (2008).

[25] A. H. Castro Neto, F. Guinea, N. M. R. Peres, K. S. Novoselov, and A. K. Geim, Rev. Mod. Phys. 81, 109 (2009).

[26] S. Das Sarma, E. H. Hwang, and W.- K. Tse, Phys. Rev. B 75, 121406(R) (2007).

[27] K. Nomura and A. H. MacDonald, Phys. Rev. Lett. 96, 256602 (2006).

[28] C. Töke, P. E. Lammert, V. H. Crespi, and J. K. Jain, Phys. Rev. B 74, 235417 (2006).

[29] V. P. Gusynin and S. G. Sharapov, Phys. Rev. B 71, 125124 (2005).

[30] V. P. Gusynin and S. G. Sharapov, Phys. Rev. Lett. 95, 146801 (2005).

[31] L. A. Falkovsky and A. A. Varlamov, Eur. Phys. J. B 56, 281 (2007).

[32] O. L. Berman, V. S. Boyko, R. Ya. Kezerashvili, A. A. Kolesnikov, and Yu. E. Lozovik, Physics Letters A 374, 4784 (2010).

[33] O. L. Berman, V. S. Boyko, R. Ya. Kezerashvili, and Yu. E. Lozovik, "Electromagnetic Wave Propagation in TwoDimensional Photonic Crystals", Chapter in the book: Wave Propagation, (InTech, Vienna, pp. 83 - 104, 2011).

[34] L. D. Landau and E. M. Lifshitz, Electrodynamics of continuous media (Second Edition, Pergamon Press, Oxford, 1984).

[35] L. A. Falkovsky and S. S. Pershoguba, Phys. Rev. B 76, 153410 (2007).

[36] L. A. Falkovsky, J. Phys.: Conf. Ser. 129, 012004 (2008).

[37] R. de L. Kronig and W. G. Penney, Proc. Roy. Soc. (London), A 130, 499 (1930).

[38] L. V. Keldysh, Sov. Phys. JETP 18, 253 (1964).

[39] J. M. Luttinger and W. Kohn, Phys. Rev. 97, 869 (1955).

[40] W. Kohn, in Solid State Physics, edited by F. Seitz and D. Turnbull, vol. 5, pp. 257- 320 (Academic, New York, 1957).

[41] E. Fermi, Notes on Quantum Mechanics (University of Chicago Press, Chicago 1995).

[42] J. D. Bjorken and S. D. Drell, Relativistic Quantum Mechanics (McGraw Hill 1964).

[43] S. Flugge, Practical Quantum Mechanics (Springer, Berlin 1999).

[44] A. A. Abrikosov, Fundamentals of the Theory of Metals (North Holland, Amsterdam, 1988). 